\title{
artigo
}

Dermatoses fúngicas na atenção básica de saúde

\section{Dermatoses fúngicas na atenção básica de saúde}

\author{
Fungal dermatoses in basic health care \\ Dermatosis fúngicas en la atención básica de salud
}

\begin{abstract}
RESUMO
As dermatoses fúngicas são infecções que ocorrem na pele ocasionada por fungos.Ademais, existem diversas infecções fúngicas, entretanto, no âmbito da atenção básica de saúde apresentam-se quatro patologias prevalentes: Malassezia. Candidíase, infecções por dermatófitos e reações dermatofíticas. 0 presente estudo teve como objetivo geral analisar como é realizado o tratamento de dermatoses fúngicas na atenção básica de saúde. Dessa forma, foi realizada a revisão bibliográfica privilegiando a análise do conteúdo de diversos artigos com resultados evidentes na literatura abarcando as bases de dados COCHRANE (Cochrane Libraly), PUBMED que contém a Medical Literature Analysis and Retrieval Sistem on-line (Medline), SCOPUS, Web of Science e Scielo. Conclui-se que conhecimento das dermatoses fúngicas permite um diagnóstico mais preciso e um tratamento mais adequado. Além de apontar as práticas na atenção básica relacionadas à dermatologia, este apanhado buscou esclarecer sobre as dermatoses fúngicas para que os profissionais interessados aprofundem seus conhecimentos.
\end{abstract}

DESCRITORES: Dermatoses; Doenças por Fungos; Atenção Básica de Saúde.

\section{ABSTRACT}

Fungal dermatoses are infections that occur on the skin caused by fungi. Furthermore, there are several fungal infections, however, within the scope of primary health care there are four prevalent pathologies: Malassezia. Candidiasis, dermatophyte infections and dermatophyte reactions. The present study aimed to analyze how fungal dermatoses are treated in primary health care. Thus, a bibliographic review was carried out, privileging the analysis of the content of several articles with evident results in the literature, covering the COCHRANE (Cochrane Libraly), PUBMED databases containing the Medical Literature Analysis and Retrieval System on-line (Medline), SCOPUS, Web of Science and Scielo. It is concluded that knowledge of fungal dermatoses allows a more accurate diagnosis and a more appropriate treatment. In addition to pointing out the practices in primary care related to dermatology, this survey sought to clarify about fungal dermatoses so that interested professionals could deepen their knowledge.

DESCRIPTORS: Dermatosis; Fungal Diseases; Primary Health Care.

\section{RESUMEN}

Las dermatosis fúngicas son infecciones que se presentan en la piel provocadas por hongos, además existen varias infecciones fúngicas, sin embargo, dentro del ámbito de la atención primaria de salud existen cuatro patologías prevalentes: Malassezia. Candidiasis, infecciones por dermatofitos y reacciones por dermatofitos. El presente estudio tuvo como objetivo analizar cómo se tratan las dermatosis fúngicas en la atención primaria de salud. Así, se realizó una revisión bibliográfica, privilegiando el análisis del contenido de varios artículos con evidentes resultados en la literatura, cubriendo las bases de datos COCHRANE (Cochrane Libraly), PUBMED que contienen el Medical Literature Analysis and Retrieval System on-line (Medline), SCOPUS, Web of Science y Scielo. Se concluye que el conocimiento de las dermatosis fúngicas permite un diagnóstico más certero y un tratamiento más adecuado. Además de señalar las prácticas en atención primaria relacionadas con la dermatología, esta encuesta buscó esclarecer sobre las dermatosis fúngicas para que los profesionales interesados pudieran profundizar sus conocimientos. DESCRIPTORES: Dermatosis; Enfermedades por hongos; Primeros auxilios.

RECEBIDO EM: 11/01/2021 APROVADO EM: 17/02/2021

\section{Eilzo Ferreira Brasileiro}

Graduação em Medicina (FCM/PB). Especialização em PSF (FIPE/PB). Médico Residente (R2) em Medicina da Família e Comunidade. Instituição: Secretaria Municipal de Saúde de João Pessoa / Faculdade de Ciências Médicas da Paraíba (FCM).

ORCID: 0000-0003-4249-6297 


\section{INTRODUÇÃO}

A Dermatologia é uma especialidade voltada para o diagnóstico e tratamento das doenças que acometem a pele. Abrange também doenças que afetam os anexos cutâneos e as mucosas. Nesse sentido, escoltada pelo progresso da Medicina, a Dermatologia apresentou nas últimas décadas, grande crescimento quantitativo e qualitativo ${ }^{1}$. Além disso, diversos estudos demonstram que as doenças de pele possuem um impacto considerável na qualidade de vida dos pacientes, destacando-se a tendência a depressão ${ }^{4}$, pois as dermatoses são apontadas como fatores de estresse, independentemente do local onde estão localizadas 5 .

Em conformidade com a Sociedade Brasileira de Dermatologia ${ }^{2}$ a incidência de dermatoses é alta e $8,8 \%$ que buscam atendimento em unidades básicas de saúde (UBS) o fizeram devido a uma dermatose fúngica. Ademais, a busca por atendimento médico em função de problemas de pele mostra-se significante atingindo cerca de $10 \%$ a $36,5 \%$ das consultas ${ }^{3}$. Contudo, verifica-se uma tendência da população em não valorizar as informações relativas aos cuidados com a pele, principalmente devido à baixa letalidade dessas patologias, subestimando sua importância como problema de saúde pública³.

As doenças dermatológicas podem ter origem infecciosa ou não. Quando a causa é infecciosa, os agressores mais comuns são bactérias, vírus e fungos ${ }^{6}$. Os principais agentes causadores das infecções fúngicas são os fungos filamentosos, denominados dermatófitos, os filamentosos não-dermatófitos e leveduras do gênero Candida e Malassezia ${ }^{7}$.

Embora pouco abordada, a temática sobre dermatoses fúngicas requer atenção em razão das eventuais consequências ao paciente na falta de tratamento e, principalmente, o impacto das mesmas na qualidade de vida.

Com isso, o presente estudo tem como objetivo geral analisar como é realizado o tratamento de dermatoses fúngicas na atenção básica de saúde. Sendo assim, apresentam-se como objetivos específicos: dissertar sobre as dermatoses fúngicas; elencar os diagnósticos e; relatar sobre os tratamentos prescritos no âmbito a atenção básica de saúde.

Com base nos argumentos apresentados, esta pesquisa se propóe buscar a resposta da seguinte questão: De que maneira a Atenção Básica de Saúde pode contribuir para melhorar a prevenção e o tratamento das dermatoses fúngicas?

\section{A Dermatologia}

é uma especialidade

voltada para o

diagnóstico e

tratamento das

doenças que

acometem a pele.

Abrange também

doenças que

afetam os anexos

\section{cutâneos e as}

\section{mucosas.}

\section{MÉTODOS}

Trata-se de estudo descritivo de abordagem qualitativa, realizado através da análise de conteúdo de diversos artigos como referencial teórico para pesquisa de dados. A pesquisa foi elaborada abarcando as bases de dados COCHRANE (Cochrane Libraly), PUBMED que contém a Medical Literature Analysis and Retrieval Sistem on-line (Medline), SCOPUS, Web of Science e SCIELO.
Foram utilizados como critérios de inclusão os artigos científicos com disponibilidade do texto completo, sem restrições de idiomas, publicados no período entre 2010 e 2020. Tal corte temporal foi estabelecido com o objetivo de fundamentar a pesquisa em dados mais recentes. Foram excluídos os artigos duplicados, aqueles que não tinham texto completo disponível e aqueles que, após a leitura, formam identificados como não relacionados à temática.

A estratégia de busca desta pesquisa utilizou-se dos seguintes descritores: "dermatoses"; "fungos"; "dermatologia”; "atenção primária”; "doenças de pele” em português e inglês. As buscas foram realizadas utilizando a junção dos MeSH Terms com os operadores booleanos OR e AND. Os estudos selecionados foram lidos, analisados, confrontados e os resultados foram apresentados de forma conceitual.

Foram encontrados 39 artigos que preencheram os critérios de elegibilidade. Destes artigos, foram selecionados 31 que abordavam sobre dermatoses causadas por fungos na atenção primária. Os dados foram apresentados de forma descritiva, visando elucidar os aspectos relevantes que envolvem o objeto de estudo.

\section{RESULTADOS}

Inicialmente, é importante enfatizar que a pele é um órgão que embora apresente resistência é passível de traumas e infecções. Nesse sentido, o estrato córneo intacto figura como uma barreira proteica localizada na superfície cutânea, que impede a entrada de patógenos ${ }^{10}$.

Com relação às infecções, essas podem ser classificadas em primárias ou secundárias, de acordo com a existência de um ponto de entrada anterior à infecção; agudas ou crônicas conforme a duração da mesma e podem também ser mono ou poli microbiana ${ }^{11}$. Já o ressecamento da pele é um fator que evita a colonização e o crescimento dos fungos, visto que o calor e umidades são essenciais para o seu desenvolvimento ${ }^{12}$.

Com relação aos fungos, é importante ressaltar que possuem grande versatilida- 
de para se adaptar e alta capacidade de contaminação. Ademais, aproximadamente 300 espécies de fungos são relatadas como patógenos de animais causando micoses, hipersensibilidades, micotoxicoses e micetismos ${ }^{8}$.

Os pacientes mais propensos a apresentarem dermatoses fúngicas são pacientes imunossuprimidos, os mais afetados devido à sua resistência à maioria dos antifúngicos disponíveis, pessoas obesas, devido ao devido ao excesso de pregas cutâneas e diabéticos ${ }^{13}$.

Em seguida serão apresentadas as principais doenças causadas por fungos na população em geral. Ademais, também será retratada a atuação das equipes de Saúde da Família na prevenção e tratamento dessas doenças.

\section{Malasseziose}

A Malasseziose é uma micose superficial, causada por leveduras do gênero Malassezia como resultado de reação inflamatória e/ou devido à hipersensibilidade a antígenos ou produtos fúngicos ${ }^{14}$. $\mathrm{O}$ gênero Malassezia abrange treze espécies de leveduras lipofílicas distintas ${ }^{15}$.

Com relação ao diagnóstico, as infecções por Malassezia geralmente são assintomáticas, podendo causar prurido ou irritação cutânea. Além disso, as lesões consistem em pequenas manchas discretas, que tendem a ser mais escuras do que a pele circundante em pacientes de pele clara e hipopigmentadas em pacientes de pele escura ${ }^{16}$.

Tais lesões costumam coalescer, formando placas amplas de diversas cores, variando do branco ao marrom claro. A raspagem das lesões produz escamas finas. Esta infecção costuma atingir a parte superior do tronco, membros superiores, virilha e abdome. A maioria das lesões exibe fluorescência amarelada sob luz de Wood ${ }^{17}$.

\section{Pitiríase Versicolor}

A Pitiríase Versicolor (PV) é uma doença causada por uma das espécies de Malassezia. Essa dermatose causa lesões que se localizam no pescoço, na face, nas costas e nos membros superiores ${ }^{16}$. As espécies de
Malassezia fazem parte da microbiota normal da pele e, geralmente são encontradas nos folículos pilosos de áreas seborreicas e com a presença de lípides, onde a levedura se transforma na sua forma parasitária como uma pseudo-hifa ${ }^{17}$.

$\mathrm{O}$ diagnóstico de PV dá-se através de cultura e análise molecular ${ }^{17}$. Em pacientes com tinea versicolor, deve-se proceder a raspagem da lesão e análise identificando a presença de pseudo-hifas e leveduras. A forma de levedura prevalece na foliculite e é facilmente observada em amostras purulentas de pústula. As biópsias destas lesões mostram a presença de leveduras ao redor e dentro dos folículos pilosos, acompanhadas de inflamação neutrofílica ${ }^{18}$. A cultura do organismo a partir do pus da foliculite mostra-se mais eficiente para o diagnóstico, visto que essas leveduras fazem parte da flora normal da pele ${ }^{17}$.

O tratamento da tinea versicolor e da foliculite causada por Malassezia envolve a aplicação de xampu à base de sulfeto de selênio. No caso da tinea versicolor, a escamação é resolvida prontamente, mas o desaparecimento das alterações pigmentares pode demorar semanas a meses ${ }^{20}$.

\section{Candidíase}

Candidíase ou candidose relaciona-se a um conjunto diferente de infecções causadas vários gêneros de Candida, sendo grande parte das infecções são causadas pela espécie Candida albicans. No entanto, espécies não $\mathrm{C}$. albicans Candida (NCAC), como Candida glabrata, Candida tropicalis e Candida parapsilosis são frequentemente identificados como patógenos humanos ${ }^{19}$.

As condições que permitem a infecção da pele causada por este fungo incluem clima quente e úmido; roupas íntimas apertadas e sintéticas; má higiene; imunodepressão por diabetes, HIV, uso de corticosteroides e outros medicamentos que debilitam o sistema imunológico; gravidez, obesidade ou uso de antibióticos ${ }^{20}$. É importante destacar que Raros casos de imunodepressão podem levar a Candida a invadir os tecidos mais profundos, bem como o sangue, e provocar uma candidíase sistêmica com risco de morte ${ }^{23}$.

A Candidíase como dermatose fúngica mostra-se como um dos casos mais comuns relatados na atenção básica de saúde. Também tende a ocorrer em áreas úmidas da pele e pode causar erupções cutâneas, escamações, coceira e inchaço ${ }^{19}$. As áreas geralmente afetadas são a mucosa da boca, a virilha, as axilas, os espaços entre os dedos das mãos e dos pés, o pênis não circuncidado, a prega cutânea sob os seios, as unhas e as pregas cutâneas na região do estômago ${ }^{21}$.

\section{Dermatófitos}

As infecções causadas por dermatófitos são chamadas de tinea. Os dermatófitos são fungos que se alimentam da proteína queratina, a qual é um componente que forma a camada externa da pele humana e compõe a estrutura dos cabelos e das unhas. Uma infecção na unha é chama$\mathrm{da}$ tinea unguium ou onicomicose. A infecção pode ocorrer em diversos locais incluindo os pés (Tinea pedis); área da barba (Tinea Barbae), no corpo (Tinea corporis), na virilha (tinea cruris); no couro cabeludo (Tinea capitis) ${ }^{25}$. Essas três espécies causadoras das dermatofitoses são de fácil disseminação e geram graves problemas à saúde pública, onde os esforços estão concentrados nas medidas de prevenção de sua difusão ${ }^{25-28}$.

Os sintomas de uma infecção causada por dermatófitos podem variar conforme a sua localização. Quando a inflamação é pequena e as áreas infectadas coçam um pouco, a borda é escamosa e um pouco elevada, com o aparecimento intermitente de placas pelo corpo. Já quando a inflamação é mais grave aparecem pontos grandes e pequenos com líquido ou uma placa inflamada no couro cabeludo com quérion ${ }^{26}$. A transmissão pode ocorrer diretamente ou indiretamente, através de materiais contaminados com escamas de pele parasitadas ${ }^{25-26}$.

O diagnóstico pode ser confirmado através de microscopia ou cultura de amostras. $\mathrm{O}$ método ideal de obtenção de amostras a partir da pele consiste na raspagem das lesões escamosas ${ }^{25-27}$. 


\section{Práticas de ESF}

O Ministério da Saúde ${ }^{29}$ elucida que a Estratégia Sáude da Familia (ESF), a qual era anteriormente denominada Programa Saúde da Família (PSF) foi criada com o intuito de reorganizar a Atenção Básica $(\mathrm{AB})$ e promover a qualidade de vida da população. Nesse contexto, a proximidade da equipe de saúde com os usuários permite uma aproximação mais adequada, o que garante maior adesão dos mesmos aos tratamentos e intervenções propostas.

A Equipe de Saúde da Família resolve grande parte dos problemas de saúde da população. Contudo, se o paciente precisar de um cuidado mais avançado, a ESF faz este encaminhamento. Assim, o atendimento pode ser realizado na unidade de saúde ou nos domicílios, facilitando o atendimento através de cadastro prévio ${ }^{29}$.

Ademais, mostra-se fundamental o papel do agente comunitário, pois por pertencer à determinada comunidade, ele conhece as questões, necessidades de saúde e cultura daquela população. Quando bem orientado, o agente será um facilitador da relação entre a equipe e a comunidade.

Nesse sentido, a ESF deve estar apta a realizar o diagnostico prévio e realizar o posterior acompanhamento das afecções prevalentes, como as infecções fúngicas. Além disso, devem ser implementadas ações voltadas para a prevenção e promoção da saúde, como determina os preceitos da $\operatorname{ESF}^{29}$.

\section{DISCUSSÃO}

As dermatoses, de modo geral representam uma alta demanda na rede básica de saúde ${ }^{2-3-6-9}$. Assim, os médicos que atuam na Atenção Primária devem estar habilitados para atender as doenças dermatológicas, diagnostica-las e adotar a melhor conduta.

Sobre as doenças de pele causadas por fungos, destaca-se que essas têm como principais agentes causadores os fungos filamentosos, denominados dermatófitos, os filamentosos não-dermatófitos e leveduras do gênero Candida e Malassezia ${ }^{6-8}$.
Observou-se que alergias, dermatoses fúngicas e acne se distribuem de forma homogênea ${ }^{2-8}$, com predomínio de micoses no sexo masculino e alergias no feminino, o que contraria os achados de Petry et. al. ${ }^{19}$, cuja pesquisa relatou a incidência de dermatoses fúngicas, especificamente pitiríase versicolor no público feminino ${ }^{3}$.

\section{Ademais, mostra- \\ se fundamental $o$ \\ papel do agente \\ comunitário, pois}

por pertencer

à determinada

comunidade, ele

conhece as questões,

necessidades de

saúde e cultura

daquela população.

Quando bem

orientado, o agente

será um facilitador da

relação entre a equipe

e a comunidade.

A Malassezia costuma ser o resultado de reação inflamatória por conta da hipersensibilidade a antígenos ou produtos fúngicos ${ }^{14-15-16-18}$. A Candida é uma levedura que reside no aparelho digestivo, no órgão genital feminino e na boca, mas não costuma causar lesões, mas em de- terminadas condições, pode causar infecções nas membranas mucosas e as áreas úmidas da pele ${ }^{19-20-21}$.

Os dermatófitos antropofílicos são a causa mais comum de infecções cutâneas por fungos. Estudos apontam características especificas para cada tipo de dermatófito conforme a região, etnia e idade, porém mais pesquisas precisam ser realizadas. Há também grande incidência de reações dermatofíticas que podem ser definidas como reações alérgicas ao fungo em áreas diversas da infecção inicial ${ }^{14-24-25-26-27}$.

Com relação à análise das dermatoses, costuma-se fazer culturas para testesmicroscópio visando identificar o fungo para determinar a melhor abordagem medicamentosa. Já em relação aos tratamentos, as infecções fúngicas geralmente são tratadas com medicamentos antimicóticos, de uso tópico e incluem cremes, géis, loções, soluções ou xampus ou podem ser administrados por via oral. ${ }^{7-24-27-28}$.

Ademais, percebe-se que é essencial realizar o treinamento contínuo dos agentes de saúde para que as equipes de saúde da família possam desenvolver um trabalho adequado de prevenção dessas doenças.

A elaboração desta análise permite aos profissionais de saúde que atuam na ESF conhecerem um pouco mais sobre essas infecções mantendo-se atentos para orientar pacientes e familiares quanto às práticas de prevenção de tratamento de dermatoses fúngicas.

\section{CONCLUSÃO}

Pode-se inferir que a equipe da ESF precisa estar atenta para orientar os pacientes em relação às dermatoses fúngicas, haja vista que são tratáveis, para que não haja reincidência. Nesse sentido, tal orientação dos profissionais de saúde junto à família pode proporcionar melhor qualidade de vida em longo prazo e reduzir a reincidência dessa patologia, ou seja, a equipe multiprofissional desempenha um papel fundamental na prevenção e controle das doenças de pele da população.

Com isso, destaca-se que o médico especialista em saúde da família está apto para a identificação e tratamento das do- 
enças mencionadas nesse estudo, porém, caso esse profissional não possua segu- rança no diagnóstico, recomenda-se o encaminhamento para um médico derma- tologista com objetivo de obter um tratamento adequado e eficiente.

\section{REFERÊNCIAS}

1. De Araújo, R. S., \& Amorim da Costa, E. M: Importância do conhecimento em dermatologia para atendimento à população ribeirinha do Rio Amazonas. Revista De Saúde. 2016; 7(2); 04-07.

2. Sociedade Brasileira de Dermatologia: Levantamento inédito mostra como está a pele dos brasileiros. Acesso em: 19 de set. 2020. Disponivel em: http://fantastico.globo.com/Jornalismo/ Fantastico/download/0,1916-1,00.doc.

3. Williams, H. C.: Epidemiology of skin disease. Rook's Textbook of Dermatology. Ninth Edition; 2016; 1-17.

4. Urasaki, M. B. M., Mandelbaum, M. H. S., \& Gonçalves, R.: Impactos psicossociais associados às manchas gravídicas. Cogitare Enfermagem; 2013; 18(4); 655-662.

5. Gascón, M. R. P., Ribeiro, C. M., Bueno, L. M. D. A., Benute, G. R. G., Lucia, M. C. S. D., Rivitti, E. A., \& Festa Neto, C.: Prevalence of depression and anxiety disorders in hospitalized patients at the dermatology clinical ward of a university hospital. Anais brasileiros de dermatologia; 2012; 87(3); 403-407.

6. Petruzzi, M. N. M. R., Cherubini, K., Salum, F. G., \& Figueiredo, M. A. Z. D.: Risk factors of HIV-related oral lesions in adults. Revista de saúde publica; 2013; 47(1); 52-59.

7. Tavares, E., Catorze, M. G., Galhardas, C., Pereira, M. J., Sá, O. B., \& Rocha, M. M. Panorama epidemiológico da infecção por dermatófitos na área de influência do Hospital Distrital de Santarém. RPDI-Revista Portuguesa de Doenças Infecciosas; 2012; 8(1).

8. REIS-GOMES, Angelita et al. Dermatopatias fúngicas: aspectos clínicos, diagnósticos e terapêuticos. Acta veterinaria brasilica; 2012; v. 6; n. 4; 272-284.

9. BERNARDES, C. A. et al. Diagnóstico e condutas dermatológicas em uma unidade básica de saúde. Revista Brasileira de Educação Médica; 2015; v. 39; n. 1; 88-94.

10. Addor, Flavia Alvim Sant'Anna; AOKI, Valeria. Barreira cutânea na dermatite atópica. Anais Brasileiros de Dermatologia; 2010; v. $85 ;$ n. $2 ; 184-194$.

11. Labrada, D. B. (2018). Comportamento e prevenção de doenças na pele na Unidade Básica de Saúde (UBS) Vila Americana. Universidade Federal de Santa Catarina; 2018; Florianópolis.

12. Sociedade Brasileira de Dermatologia. Micose. Acesso em: 01 de set. 2020. Disponivel em: https://www.sbd.org.br/dermatologia/pele/doencas-e-problemas/micose/14/

13. Peman, J., \& Salavert, M. Invasive fungal disease due to Scedosporium, Fusarium and mucorales. Revista iberoamericana de micologia; 2014; 31(4); 242-248.

14. Meredith, A. Dermatoses. In BSAVA Manual of Rabbit Medicine; 2014; BSAVA Library; 255-263.

15. Neves, J. J. A., Paulino, A. O., Vieira, R. G., Nishida, E. K., \& Coutinho, S. D. A. The presence of dermatophytes in infected pets and their household environment. Arquivo Brasileiro de Medicina
Veterinária e Zootecnia; 2018; 70(6); 1747-1753.

16. Rasi, A., Naderi, R., Behzadi, A. H., Falahati, M., Farehyar, S., Honarbakhsh, Y., \& Akasheh, A. P. Malassezia yeast species isolated from Iranian patients with pityriasis versicolor in a prospective study. Mycoses; 2010; 53(4); 350-355.

17. Petry, V., Tanhausen, F., Weiss, L., Milan, T., Mezzari, A., \& Weber, M. B. Identificação de espécies de malassésia na pitiríase versicolor em um serviço de dermatologia do sul do Brasil. Anais Brasileiros de Dermatologia; 2011; 86(4); 803-806.

18. Framil, V. M. D. S., Melhem, M. S., Szeszs, M. W., Corneta, E. C., \& Zaitz, C. (2010). Pitiríase versicolor: isolamento e identificação das principais espécies de Malassezia. Anais Brasileiros de Dermatologia; 2010; 85(1); 111-114.

19. Silva, S., Negri, M., Henriques, M., Oliveira, R., Williams, DW, \& Azeredo, J. Candida glabrata, Candida parapsilosis e Candida tropicalis: biologia, epidemiologia, patogenicidade e resistência antifúngica. FEMS microbiology reviews; 2012; 36 (2); 288-305.

20. Calderone, RA, \& Clancy, CJ (Eds.). Candida e candidíase. American Society for Microbiology Press; 2011.

21. Mayer, FL, Wilson, D., \& Hube, B. Mecanismos de patogenicidade de Candida albicans. Virulence. 2013; 4 (2); 119-128.

22. De Rossi, T., Lozovoy, MAB, da Silva, RV, Fernandes, EV, Geraldino, TH, Costa, IC, \& Felipe, I. Interações entre Candida albicans e hospedeiro. Semina: Ciências Biológicas e da Saúde; 2011; 32 (1); $15-28$.

23. Giolo, M. P., \& Svidzinski, T. I. E. Fisiopatogenia, epidemiologia e diagnóstico laboratorial da candidemia. Jornal Brasileiro de Patologia e Medicina Laboratorial. 2010; 46(3); 225-234.

24. Bitencourt, R. P. Ocorrencia de Candida em alicates de cutícula em centros de estética; 2015.

25. Summerbell, R. C. Trichophyton, Microsporum, Epidermophyton, and agents of superficial mycoses. American Society of Microbiology; In Manual of Clinical Microbiology; 2011; 10th Edition; 1919-1942.

26. Padhye, AA e Summerbell, RC. Os dermatófitos. Microbiologia e infecções microbianas de Topley \& Wilson; 2010.

27. Abd Elmegeed, ASM, Ouf, SA, Moussa, TA, \& Eltahlawi, SMR. Dermatófitos e outros fungos associados em pacientes atendidos em alguns hospitais no Egito. Revista Brasileira de Microbiologia; 2015; 46 (3); 799-805.

28. Dalla Lana, D. F., Batista, B. G., Alves, S. H., \& Fuentefria, A. M. Dermatofitoses: agentes etiológicos, formas clínicas, terapêutica e novas perspectivas de tratamento. Clinical and biomedical research; 2016; Vol. 36; n. 4; 230-241.

29. Brasil. Ministério da Saúde. Estratégia Saúde da Familia. Acesso em: 19 de set. 2020. Disponivel em: http://www.saude.gov.br/ acoes-e-programas/saude-da-familia/sobre-o-programa 